\title{
NÍVEL DE ATIVIDADE FÍSICA EM PESSOAS COM HIPERTENSÃO ARTERIAL SISTÊMICA
}

\author{
LEVEL OF PHYSICAL ACTIVITY IN PEOPLE WITH \\ SYSTEMIC ARTERIAL HYPERTENSION
}

\section{NIVEL DE ACTIVIDAD FÍSICA EN PERSONAS CON HIPERTENSIÓN ARTERIAL SISTÉMICA}

\author{
Cleise Cristine Ribeiro Borges Oliveira ${ }^{1}$ \\ Maiara da Silva Brandão Rodrigues ${ }^{2}$ \\ Carla Tatiane Oliveira Silva ${ }^{3}$ \\ Cláudia Silva Marinho Antunes Barros ${ }^{4}$ \\ Elieusa e Silva Sampaio ${ }^{5}$ \\ Cláudia Geovana da Silva Pires ${ }^{6}$
}

Como citar este artigo: Oliveira CCRB, Rodrigues MSB, Silva CTO, Barros CSMA, Sampaio ES, Pires CGS. Nível de atividade física em pessoas com hipertensão arterial sistêmica. Rev baiana enferm. 2019;33:e-33648.

\begin{abstract}
Objetivo: avaliar o nível de atividade física entre pessoas com hipertensão arterial sistêmica. Método: estudo descritivo, de abordagem quantitativa, realizado em um multicentro de saúde da cidade de Salvador, Bahia, com participação de 220 pessoas com diagnóstico médico de hipertensão arterial sistêmica. Investigou-se nível de atividade física dos participantes no trabalho, no trajeto de deslocamento, nas tarefas domésticas e no tempo livre com base no Questionário Internacional de Atividade Física e na classificação: muito ativos, ativos, irregularmente ativos e sedentários. Realizou-se análise descritiva para caracterizar a população do estudo. Resultados: predomínou comportamento sedentário em todos os indicadores de atividade física avaliados pelo Questionário Internacional de Atividade Física. Conclusão: conhecendo a gravidade da hipertensão arterial sistêmica e a necessidade da atividade física para prevenção e controle, é necessária maior vigilância e mais estratégias para, respectivamente, melhor compreender a magnitude e modificar o comportamento sedentário que predomina em pessoas com essa doença.
\end{abstract}

Descritores: Hipertensão. Comportamento Sedentário. Estilo de Vida. Fatores de Risco.

Objective: to evaluate the level of physical activity among people with systemic arterial hypertension. Method: a descriptive study with a quantitative approach, conducted at a health center in the city of Salvador, Babia, with the participation of 220 people with a medical diagnosis of systemic arterial hypertension. The participants' physical activity level at work, commuting to work, household chores and free time was investigated based on the International Physical Activity Questionnaire and on the following classification: very active, active, irregularly active and sedentary. A descriptive analysis was performed to characterize the study population. Results: a sedentary behavior predominated in all physical activity indicators evaluated by the International Physical Activity Questionnaire. Conclusion: knowing the severity of systemic arterial bypertension and the need for physical activity for prevention

\footnotetext{
Enfermeira. Especialista em Enfermagem e Centro Cirúrgico. Universidade Federal da Bahia. Salvador, Bahia, Brasil. cleisecristine@gmail.com https://orcid. org/0000-0002-0946-5627

Estudante de Enfermagem. Universidade Federal da Bahia. Salvador, Bahia, Brasil. https://orcid.org/0000-0003-3269-1953

Enfermeira. Universidade Federal da Bahia, Salvador, Bahia, Brasil. https://orcid.org/0000-000 I-7824-9998

Enfermeira. Doutora em Ciências na área de concentração em Biotecnologia e Medicina Investigativa. Professora da Universidade Federal da Bahia. Salvador, Bahia, Brasil. https://orcid.org/0000-0002-0597-8I 26

5 Enfermeira. Doutora em Medicina. Professora da Universidade Federal da Bahia. Salvador, Bahia, Brasil.

6 Enfermeira. Doutora em Enfermagem. Professora da Universidade Federal da Bahia. Salvador, Bahia, Brasil. https://orcid.org/0000-000 I-9309-28 I0
} 
and control requires greater surveillance and more strategies, respectively, to better understand the magnitude and modify the sedentary behavior that predominates in people with this disease.

Descriptors: Hypertension. Sedentary Behavior. Lifestyle. Risk Factors.

Objetivo: evaluar el nivel de actividad física en personas con hipertensión arterial sistémica. Método: estudio descriptivo con enfoque cuantitativo realizado en un multicentro de salud de la ciudad de Salvador, Babia, con la participación de 220 personas con diagnóstico médico de hipertensión arterial sistémica. Se investigó el nivel de actividad fisica de los participantes en el trabajo, en los viajes para ir y volver del trabajo, en las tareas domésticas y en el tiempo libre, sobre la base del Cuestionario Internacional de Actividad Física y de la siguiente clasificación: muy activos, activos, activos irregularmente y sedentarios. Se efectuó un análisis descriptivo para caracterizar a la población del estudio. Resultados: se registró un predominio del comportamiento sedentario en todos los indicadores de la actividad física evaluados por el Cuestionario Internacional de Actividad Fúsica. Conclusión: al conocer la gravedad de la bipertensión arterial sistémica y la necesidad de la actividad física para su prevención y control, se necesitan, respectivamente, más supervisión y más estrategias para comprender mejor la magnitud y modificar el comportamiento sedentario que predomina en las personas que padecen esta enfermedad.

Descriptores: Hipertensión. Comportamiento Sedentario. Estilo de Vida. Factores de Riesgo.

\section{Introdução}

As doenças crônicas não transmissíveis (DCNT) são responsáveis pela principal causa de mortalidade global (63\%). Sua prevalência cresce de forma assustadora na população. Dentre elas, a Hipertensão Arterial Sistêmica (HAS) ocorre com maior frequência, sendo estimada em torno de 1,13 bilhão de pessoas no mundo, isto é, 30 a $45 \%$ da população adulta apresentam esta doença ${ }^{(1-2)}$.

Apesar dos avanços no diagnóstico e tratamento, dentre as DCNT, a HAS ainda é responsável pela maior parte da mortalidade e incapacidades geradas mundialmente, sendo causadora de 54\% das doenças cerebrovasculares, $47 \%$ das doenças isquêmicas do coração e assumindo $13 \%$ das mortes ocorridas a cada ano. Estima-se que, em 2025, a prevalência de HAS no mundo terá um aumento de 15 a 20\%, podendo chegar a atingir uma população de 1,5 bilhão de pessoas ${ }^{(2)}$.

No Brasil, a prevalência da HAS é de 31,0\%, atinge $32,5 \%$ dos adultos e mais de 60\% dos idosos, contribuindo com 50\% das mortes ocasionadas por doença cardiovascular ${ }^{(3)}$. Estima-se que a prevalência geral no Brasil alcançará 40\% em $2025^{(4)}$. Na capital da Bahia, Salvador, o percentual de adultos que referem ser portadores de HAS é de 27,3\%. Nessa mesma cidade, tem-se um percentual de mulheres portadoras de HAS equivalente a 28,7\%; quanto aos homens, esse percentual alcança $21,1 \%{ }^{(5)}$.

Essa doença está diretamente vinculada a distúrbios do metabolismo, alterações nas funções e estruturas dos órgãos-alvo e é agravada por diversos fatores de risco ${ }^{(3)}$. É muito rara a ocorrência da HAS de forma isolada. Ela ocorre mais frequentemente associada a outros fatores de risco para doenças cardiovasculares (DCV) ${ }^{(2)}$.

Há evidências na literatura científica sobre a existência de diversos fatores de risco que concorrem para $\operatorname{HAS}^{(1)}$. Estudos chamam a atenção cada vez mais para a relação existente entre as disparidades socioeconômicas e a prevalência de HAS $^{(6-8)}$. Alertam que as desigualdades no âmbito socioeconômico podem implicar em outras diferenças quanto ao acesso aos serviços de saúde e ao recebimento de orientações e cuidados necessários para sua prevenção e controle ${ }^{(9-10)}$.

Além dos fatores socioeconômicos, a inatividade física constitui o quarto fator de risco mais prevalente na população e é responsável pela segunda causa de morte no mundo, considerada um dos maiores problemas de saúde pública. Causa, em média, 3,2 milhões de mortes em cada ano ${ }^{(3,11)}$. A inatividade física constitui uma tendência do estilo de vida que contribui para a manutenção das DCV como a principal causa de 
mortalidade no Brasil desde o final da década de 1960. Isso decorre da diminuição do gasto energético propiciado pelo fator comportamental ${ }^{(12)}$.

Os adultos que se apresentam em condição de inatividade física possuem um risco de mortalidade aumentado em torno de 20 a 30\% por todas as causas em comparação aos que realizam semanalmente no mínimo 150 minutos de atividade física ${ }^{(6)}$. Estudos apontam que 46\% da população adulta encontra-se insuficientemente ativa ${ }^{(3)}$.

Em situação complementar a essas informações, é evidenciado percentual de 31,8\% no conjunto das 27 capitais brasileiras do percentual de adultos $\geq 18$ anos que acumulam, no mínimo, 150 minutos de atividade física moderada por semana no tempo livre. Na população de Salvador, o percentual de adultos $\geq 18$ anos que acumula esse tempo mínimo de atividade física moderada semanal no tempo livre é de 14,5\%. Quando avaliados adultos com prática insuficiente de atividade física nessa cidade, o percentual encontrado é de $41,5 \%{ }^{(5)}$.

A adoção de melhores hábitos de vida, como a prática de atividade física regular, é considerada atualmente, nas discussões mundiais, como um método efetivo para prevenção de diversas doenças ${ }^{(2)}$. Dentre elas, contribui para prevenir e tratar DCNT, tais como DCV, Diabetes Mellitus, acidente vascular cerebral (AVC), excesso de peso e obesidade, bem como a HAS, promovendo saúde, qualidade de vida e bem-estar ${ }^{(13)}$.

O desenvolvimento regular de atividade física, mesmo em menor intensidade, pode contribuir na prevenção e controle da HAS, sendo consequentemente benéfica para a diminuição em 20\% do índice da mortalidade mundial ${ }^{(14)}$. O nível de atividade física regular possibilita a redução do risco de desenvolver HAS em cerca de $30 \%$ das pessoas, quando comparada à condição de inatividade física, pois o aumento implica diretamente na redução da pressão arterial $(\mathrm{PA})^{(3)}$.

Indivíduos que não realizam atividade física regularmente possuem duas vezes mais chances de apresentar complicações coronarianas quando comparados a indivíduos que realizam atividade física regularmente. Do mesmo modo, há probabilidade maior de apresentarem IAM, principalmente na presença de outros fatores de risco associados como a $\mathrm{HAS}^{(15)}$.

Frente a esse contexto, o presente estudo apresenta como objetivo avaliar o nível de atividade física entre pessoas com hipertensão arterial sistêmica.

\section{Método}

Trata-se de um estudo descritivo, de abordagem quantitativa. Constitui um recorte temático de uma pesquisa matriz desenvolvida na Universidade Federal da Bahia, intitulada "O impacto dos Determinantes Sociais em Saúde na Prevenção e Controle dos Fatores de Risco Cardiovascular em Pessoas Hipertensas".

A pesquisa foi realizada no período de julho a dezembro de 2017, em um multicentro de Saúde de um bairro populoso da cidade de Salvador, de origem e cultura afrodescendentes, o qual atende pessoas que frequentam o Serviço de Doenças Crônicas do Sistema Único de Saúde para o atendimento da clientela hipertensa e diabética. O serviço atua de forma complementar à rede de atenção básica e também oferece atendimento médico nas seguintes especialidades: clínica geral, ortopedia, pediatria, ginecologia, oftalmologia, endocrinologia entre outras. Além disso, oferece atendimento de nutricionista, psicólogo e assistente social.

A população do estudo correspondeu a pessoas de ambos os sexos com diagnóstico médico de Hipertensão Arterial Sistêmica (CID: I-10) atendidas no multicentro de saúde abordado. Com o objetivo de encontrar a proporção de pessoas acima de 18 anos atendidas no multicentro de saúde, que apresentavam diagnóstico de HAS, calculou-se a amostra aleatória simples sem reposição, utilizando o software STATA na sua versão 11. Para isso, foi adotada a prevalência de 25,7\% da HA em pessoas acima de 18 anos com base nos resultados revelados pela Pesquisa Vigitel no ano de $2014^{(5)}$, admitido um erro amostral equivalente a $5 \%(\mathrm{~d}=0,05)$, sob o nível de confiança de 95\%. O denominador utilizado foi o total de indivíduos cadastrados no 
Sistema de Cadastramento e Acompanhamentos de Hipertensos e Diabéticos do Programa Nacional de Hipertensão e Diabetes Mellitus (Hiperdia). Mediante cálculo, o tamanho da amostra foi de 220 pessoas com diagnóstico médico de HAS.

Como critérios de inclusão na pesquisa, foram definidos: pessoas com diagnóstico médico de HAS que frequentavam o multicentro de saúde para realização de consultas e retirada de medicamentos por meio de receita, no período da coleta de dados, e que apresentavam idade igual ou superior a 18 anos. Os critérios de exclusão foram assim definidos: gestantes, pessoas com sequelas motoras e acometidas por distúrbios psiquiátricos e/ou cognitivos por meio de diagnóstico prévio. Os participantes do estudo foram selecionados de forma aleatória, enquanto aguardavam consulta no programa Hiperdia e/ou dispensação de medicamentos na farmácia.

Os dados foram coletados por meio de entrevista estruturada da pesquisa matriz, a qual foi guiada por um instrumento com questões fechadas e semiestruturadas. No instrumento, foram investigados dados referentes aos determinantes sociais de saúde: sexo, idade em anos, raça/cor autodeclarada, responsável ou chefe da família, nível de escolaridade do responsável ou chefe da família, renda mensal da família, quantidade de pessoas dependentes dessa renda, local e condições de moradia, bem como a situação conjugal.

O Questionário Internacional de Atividade Física (International Physical Activity Questionnary - IPAQ) foi utilizado para a investigação dos dados sobre atividade física. Este questionário investiga o nível de atividade física desenvolvida no trabalho, no trajeto de deslocamento, nas tarefas domésticas e no tempo livre com base nos seguintes itens: frequência (dias da semana), duração (tempo por dia) e intensidade (leve, moderada e vigorosa). De acordo com o questionário, as pessoas são classificadas como: muito ativas, ativas, irregularmente ativas e sedentárias. Estas duas últimas classificações são consideradas de risco para o indivíduo.

Os dados foram codificados e digitados no software estatístico Statistical Package for the Social Sciences (SPSS), versão 21.0, para tratamento e geração dos resultados. Foram realizadas análises descritivas, para caracterizar a população do estudo. As variáveis estudadas foram apresentadas descritivamente em tabelas contendo frequências absolutas (n) e relativas (\%).

A pesquisa matriz que originou este estudo possui Parecer de Aprovação no ${ }^{-1.182 .032, ~ d e ~}$ 6/5/2015, do Comitê de Ética em Pesquisa (CEP) da Escola de Enfermagem da Universidade Federal da Bahia. Foram respeitados os princípios éticos aprovados pela Resolução no 466, de dezembro de 2012, Resolução n⿳0 510, de abril de 2016, e Resolução no 580, de março de 2018, todas do Conselho Nacional de Saúde, que regulamentam as pesquisas que envolvem seres humanos. Foram igualmente respeitados os quatro princípios básicos da Bioética de não maleficência, justiça, beneficência e autonomia.

Todos os participantes da pesquisa foram previamente informados a respeito dos objetivos da pesquisa, justificativas, assim como benefícios e riscos que envolviam sua participação no estudo. Foi obtida a anuência de cada participante.

\section{Resultados}

A amostra foi constituída por 220 pessoas com HA. Houve supremacia do sexo feminino (78,6\%); a idade média foi de 59,4 anos (DP = $11,3)$, predominou a faixa etária maior ou igual a 60 anos (53,6\%); de cor preta e parda (91,4\%); ensino médio completo (55,0\%); renda familiar mensal de um a dois salários mínimos (48,6\%); e estando com companheiro(a) (77,7\%).

Em relação ao nível de atividade física, de acordo com os critérios do IPAQ, predominou o comportamento sedentário nas seções: trabalho (93,6\%), meio de transporte (84,1\%), atividades de casa $(75,5 \%)$, lazer $(97,3 \%)$. No tempo gasto sentado, as pessoas foram classificadas, em maior proporção, como insuficientemente ativas (43,6\%), conforme demonstra Tabela 1. Portanto, de acordo com os percentuais encontrados para sedentarismo, houve predomínio de comportamento sedentário/insuficientemente ativo em todos os indicadores de atividade física. 
Tabela 1 - Nível de atividade física de pessoas com hipertensão arterial sistêmica. Salvador, Bahia, Brasil - jul.-dez. 2017 (N=220)

\begin{tabular}{l|c|c|c}
\hline \multirow{2}{*}{ Indicadores de atividade física } & \multicolumn{3}{|c}{ Classificação do nível de atividade física } \\
\cline { 2 - 4 } & $\begin{array}{c}\text { Ativo } \\
\text { n (\%) }\end{array}$ & $\begin{array}{c}\text { Sedentário } \\
\text { n (\%) }\end{array}$ & $\begin{array}{c}\text { Insuficientemente ativo } \\
\text { n (\%) }\end{array}$ \\
\hline Atividade física no trabalho & $14(6,4)$ & $206(93,6)$ & - \\
Atividade física como meio de transporte & $4(1,8)$ & $185(84,1)$ & $31(14,1)$ \\
Atividade física em casa & $31(14,1)$ & $166(75,5)$ & $23(10,5)$ \\
Atividade física no lazer & - & $214(97,3)$ & $6(2,7)$ \\
Tempo gasto sentado & $34(15,5)$ & $90(40,9)$ & $93(43,6)$ \\
\hline
\end{tabular}

Fonte: Elaboração própria.

Nota: Sinal convencional utilizado:

- Dado numérico igual a zero não resultante de arredondamento.

\section{Discussão}

A análise das características socioeconômicas da população mostrou predomínio de mulheres. Nota-se, na literatura científica, que, apesar da diminuição do número de mulheres com HAS nos últimos anos, a prevalência autorreferida na população mundial é maior em mulheres quando comparado a homens. As mulheres estão mais inclinadas ao aumento do risco de eventos cardiovasculares em decorrência de elevação da PA. No entanto, é curioso destacar que maior nível de conscientização a respeito da HAS está sendo observado em mulheres, bem como o atendimento nos serviços de saúde é mais prevalente no sexo feminino ${ }^{(16)}$.

Diferente da realidade brasileira, nos Estados Unidos, pesquisa desenvolvida com o intuito de avaliar as tendências no controle da HAS de 1999 a 2016 por sexo, constatou predomínio de HAS no sexo masculino, referindo prevalência decrescente durante esses anos, enquanto, para as mulheres, não houve mudança na prevalência da doença ${ }^{(17)}$.

No que tange à faixa etária, predominaram pessoas com idade superior a 60 anos que mais padecem de HAS. Corrobora este resultado, estudo desenvolvido na África, que também apresentou maior percentual de pessoas com HAS nessa faixa etária e atribuiu ao envelhecimento a condição de fator contribuinte no aumento da carga dessa doença no país ${ }^{(18)}$. Os pacientes mais acometidos por HAS são idosos ${ }^{(19)}$.
Esse predomínio de idosos com HAS pode estar relacionado com o aumento da resistência dos vasos sanguíneos e consequente enrijecimento arterial que ocorre com o avançar da idade, apresentando início entre os 20 e 30 anos, intensificando-se entre os 30 e $50 \operatorname{anos}^{(20)}$. Essa base biológica para elevação da PA em idosos, devido ao enrijecimento arterial, implica em diversas complicações para a estrutura e função do coração. Além disso, com o avançar da idade, existe uma tendência à redução da função renal, devido ao declínio de enzimas plasmáticas importantes, que compromete os mecanismos homeostáticos e prejudica o controle da PA sanguínea ${ }^{(18)}$.

No que se refere à característica raça/cor autorreferida, constatou-se predomínio da raça negra/cor preta e parda. Esperava-se esse resultado, por tratar-se de pesquisa desenvolvida em bairro de origem e cultura afrodescendente. Vê-se, em estudo realizado no ano de 2016, predomínio de HAS na população parda ${ }^{(20)}$. Em outro estudo, realizado no ano de 2017, também foi constatada prevalência de HAS em indivíduos com cor da pele preta ${ }^{(21)}$.

Essa doença é conhecida mundialmente como um dos principais problemas enfrentados no âmbito da saúde pública da população negra, principalmente no Brasil, por ser a segunda maior nação de afrodescendentes e, por isso, tem a HAS como uma das doenças que mais preocupa $^{(21)}$. Nos Estados Unidos (EUA), a prevalência de HAS é 50\% maior em pessoas afro-descendentes. Além disso, nesse grupo étnico, é 
menos provável o alcance de nível de atividade física regular como prevenção e controle das $\mathrm{DCV}^{(22)}$.

Outro estudo desenvolvido nos EUA corrobora esses dados, ao relatar que pessoas afrodescendentes são mais propensas a apresentarem HAS e em idade mais jovem do que pessoas de outros grupos étnicos, além de serem mais propensas a apresentar PA fora de controle. Esses fatores contribuem para elevação da taxa de mortalidade decorrente de DCV em pessoas afrodescendentes. Além disso, esse mesmo estudo afirma que a discriminação racial constitui um fator de risco para essas pessoas, aspecto que engloba comportamentos do racismo, incluindo ações vivenciadas em diversos locais sociais que vão desde o distanciamento social à agressão, fazendo-os viver em constante vigilância, chamada "estratégia de enfrentamento vigilante", desencadeando respostas psicológicas e cardiovasculares condicionantes ao estresse ${ }^{(23)}$.

No que tange aos níveis de escolaridade nessa população, predominou o ensino médio completo, seguido de ensino fundamental incompleto, isto é, bem poucas foram as pessoas que apresentaram nível superior de escolaridade. Esse resultado é frequentemente encontrado nas pesquisas com pessoas que padecem de $\operatorname{HAS}^{(14,21)}$.

É visto em outro estudo que 79\% dos indivíduos com HAS apresentam analfabetismo ou ensino fundamental incompleto. Também foi visto na literatura, em pesquisa de tendência temporal com pessoas hipertensas, o predomínio de ensino fundamental incompleto, seguido de fundamental completo e médio completo ${ }^{(21)}$, reforçando, mais uma vez, a relação do baixo nível de escolaridade com o desenvolvimento e descontrole da doença.

Estudos recentes têm mostrado que as populações com menor escolaridade possuem menos acesso aos serviços de saúde e recebem cuidados preventivos com menor intensidade, e esses fatores podem sugerir dificuldade de compreensão das informações de saúde e baixo nível de conhecimento sobre o processo saúde-doença, patologias crônicas, como a HAS, e seus respectivos modos de prevenção e tratamento, contribuindo para o descontrole dessa doença ${ }^{(10)}$.

Com relação à renda dos participantes da pesquisa, predominaram pessoas com renda mensal de 1 a 2 salários mínimos, seguida de renda menor que 1 salário mínimo; poucos apresentaram renda superior a 2 salários mínimos. Esses resultados corroboram outros estudos, havendo uma variação em relação ao número de salários mínimos mensais, como renda menor que um salário mínimo ${ }^{(20)}$ e renda menor que três salários mínimos ${ }^{(21)}$, o que sugere vulnerabilidade quanto à condição socioeconômica em grupos de hipertensos.

Estudo recente realizado na África também discorre sobre a elevada prevalência de HAS nesse continente. Ressalta que aspectos socioeconômicos, como baixos níveis de escolaridade e renda, contribuem para o desenvolvimento, baixa detecção e pouco controle dessa doença, já que interferem na forma como os indivíduos conduzem a vida e adotam os hábitos diários. Famílias pobres tendem a ter menos consciência a respeito de prevenção e controle da HAS e, portanto, tendem a alcançar piores resultados quanto às complicações decorrentes dessa doença ${ }^{(18)}$.

No que concerne à situação conjugal, evidenciou-se que a maioria das pessoas com HAS tem companheiro(a). Sugere-se que, com o passar da idade, as pessoas tendem a estreitar laços afetivos e resolvem estabelecer um relacionamento mais sério.

Em países do Sul Asiático foram desenvolvidos estudos que apontaram a relação existente entre as diferenças socioeconômicas e a prevalência de HAS, pois observaram que pessoas de baixa renda, com menor escolaridade, que moravam em bairros desfavorecidos, apresentaram maior probabilidade de desenvolver complicações e morrer em decorrência dessa doença. Já as pessoas que padeciam de HAS e possuíam maior nível socioeconômico apresentaram relação inversa, ao manifestarem melhor estado de saúde ${ }^{(6-8)}$. Desse modo, pode-se entender que as diferenças socioeconômicas podem direcionar os indivíduos a apresentar baixo acesso a 
cuidados de saúde, educação, trabalho e lazer, fatores que geram danos graves à saúde ${ }^{(9)}$.

Estudo realizado na Coreia do Sul conjectura que a condição socioeconômica menos favorecida pode interferir nos hábitos de vida adotados pelas pessoas, propiciando o comportamento alimentar inadequado, o estímulo ao sedentarismo, bem como outros hábitos inadequados que dificultam a adoção de um estilo de vida saudável, desenvolvendo no indivíduo distúrbios do metabolismo e doenças como a HAS. Além disso, as populações menos favorecidas socioeconomicamente possuem menor acesso aos serviços de saúde e tendem a apresentar menor probabilidade de receber orientações e cuidados preventivos apropriados ${ }^{(10)}$.

Destaca-se, neste estudo, o predomínio do comportamento sedentário/insuficientemente ativo nas pessoas com HAS, no tocante a todos os indicadores de atividade física avaliados. Observou-se também que prevaleceram padrões comportamentais inadequados em países como China e EUA, os quais são fatores de risco associados ao desenvolvimento de DCVs, como a $\mathrm{HAS}^{(24)}$. Importante salientar que indivíduos que mantêm comportamento sedentário possuem duas vezes mais possibilidade de desenvolver DCV quando comparados a indivíduos que praticam atividade física regularmente. Ainda mais preocupante: pessoas sedentárias e com diagnóstico de HAS possuem maior chance de complicações, principalmente na presença de fatores de risco cardiovasculares associados, como fatores comportamentais ${ }^{(15)}$.

Situações relacionadas ao estilo de vida, como a inatividade física/sedentarismo, constituem fatores de risco que podem resultar em consequências graves para os indivíduos. Entretanto, a modificação nessa condição pode reduzir o risco de desenvolvimento e descontrole das DCVs, como a HAS, tendo em vista que a principal conduta para prevenir e controlar essa doença é a adoção de comportamentos saudáveis. A prática de atividade física constitui um comportamento saudável que produz benefícios importantes para a saúde, como a redução de 5 a $7 \mathrm{mmHg}$ da PA e sistólica em pessoas com HAS ${ }^{(25)}$.
É recomendada, para as pessoas com HAS, a realização de, no mínimo, $30 \mathrm{~min}$ de atividade física moderada diariamente, de forma contínua ou acumulada, sendo desenvolvida em pelo menos 5 dias da semana, podendo ser realizada nas diversas modalidades, como andar, correr e nadar $^{(4)}$. Entretanto, mesmo diante dessas orientações mundiais, o que se observou é que não eram seguidas pelos indivíduos com HAS estudados. Estes mantinham hábitos sedentários em todos os domínios da atividade física e, por conseguinte, apresentavam maior chance de agravar a doença e a morbimortalidade ${ }^{(14)}$.

No que concerne ao nível de atividade física da população brasileira, identifica-se que 44,1\% não a realiza de forma suficiente. Esse percentual é maior no sexo feminino(5). Nessa população, 46\% dos indivíduos não chegaram a atingir 150 minutos de atividade física na semana, incluindo atividade do trabalho, em casa, no lazer e no deslocamento. Registraram-se diferenças percentuais, conforme a faixa etária, sendo os idosos os que apresentaram o maior percentual, equivalente a $62,7 \% \%^{(3)}$.

Desde o ano de 1990, o Brasil vem implantando políticas de intervenção para promoção e incentivo de atividade física no intuito de monitorar e diminuir e/ou minimizar os fatores de ricos cardiovasculares. Em 2011 foram consolidadas ações propostas pelo plano de ação para enfrentar doenças não transmissíveis, nas quais estão incluídos objetivos a serem alcançados até 2022, dentre eles a prevenção de HAS, estabelecida como uma das prioridades da atenção primária ${ }^{(12)}$.

Observa-se, entre os indivíduos que apresentam nível de atividade física suficiente, o favorecimento na redução do risco de desenvolver HAS em cerca de 30\% quando comparados a indivíduos em condição de inatividade física, pois esse aumento implica diretamente na redução da $\mathrm{PA}^{(3)}$.

Diante das complicações e da mortalidade decorrentes da HAS, observa-se que, de fato, são necessários olhares mais aprofundados por parte dos responsáveis pela formulação de políticas públicas, para conhecer de perto a realidade de cada indivíduo dentro de seus respectivos 
locais de moradia, para que, com base nesse conhecimento, sejam avaliadas, implantadas e implementadas estratégias de saúde pautadas na realidade, no intuito de promover redução das desigualdades no âmbito da saúde ${ }^{(6)}$.

Além disso, é preciso que os órgãos públicos invistam em estruturas adequadas no ambiente físico das localidades, para propiciar a prática de atividade física pela população e facilitar o desenvolvimento regular de caminhadas, corridas e ciclismo, bem como outras formas de atividade física não motorizadas, como natação, além de oportunizar a realização de esportes, atividades de lazer e recreação, que favorecem uma vida ativa a todas as faixas etárias ${ }^{(11)}$.

Este estudo apresentou algumas limitações, como a amostra pequena, e por se tratar de um grupo que frequenta o serviço de saúde numa comunidade de origem e cultura predominantemente afrodescendentes. Esses aspectos podem ter contribuído para a amostra apresentar a maioria com raça negra/cor preta e parda, dificultando maiores considerações quanto ao fator raça/cor no estudo.

\section{Conclusão}

O nível de atividade física sedentário/insuficientemente ativo predominou entre pessoas com HAS que participaram deste estudo. Destaca-se que o sedentarismo preponderou em todos os domínios da atividade física: no trabalho, no transporte, em casa, no lazer e no tempo gasto sentado. Isso reflete a dificuldade de adesão ao estilo de vida saudável por parte dos indivíduos com essa doença e, consequentemente, maior risco de provocar o descontrole da doença. Portanto, é importante que os serviços de saúde estimulem a prática de atividade física, o que requerer dos profissionais atenção especial, por meio de atividades educativas de promoção da saúde da população com HAS.

Apesar das limitações apontadas, o estudo permitiu conhecer e avaliar o nível de atividade física das pessoas com HAS e constatar comportamentos inadequados quanto a essa prática, que podem apresentar potencial risco para a gravidade da doença. Portanto, os resultados deste estudo foram pertinentes para ressaltar a necessidade de maior vigilância e de mais estratégias de intervenção entre pessoas com HAS, para melhor compreender a magnitude e estimular a prática de atividade física regular com vistas a modificar o comportamento sedentário/insuficientemente ativo que predomina em pessoas com esta doença.

Além disso, os resultados podem estimular novos estudos científicos sobre a temática, com delineamentos mais aprofundados para essa investigação, e também estudos randomizados, com amostras maiores, para melhor definir fatores que estejam associados ao comportamento sedentário/insuficientemente ativo observado em individuos com HAS. Permitirão igualmente fortalecer o entendimento sobre a necessidade de ações preventivas e de controle dos fatores de risco cardiovasculares nas políticas públicas de saúde, por meio de programas de educação em saúde que englobem atividades pertinentes e frequentes para o estímulo à adoção e estilo de vida mais saudável entre pessoas com HAS, abrangendo a saúde de todas as populações e suas diversas características socioeconômicas.

\section{Colaborações:}

1 - concepção, projeto, análise e interpretação dos dados: Cleise Cristine Ribeiro Borges Oliveira, Elieusa e Silva Sampaio e Cláudia Geovana da Silva Pires;

2 - redação do artigo e revisão crítica relevante do conteúdo intelectual: Cleise Cristine Ribeiro Borges Oliveira, Maiara da Silva Brandão Rodrigues, Carla Tatiane Oliveira Silva, Cláudia Silva Marinho Antunes Barros, Elieusa e Silva Sampaio e Cláudia Geovana da Silva Pires;

3 - aprovação final da versão a ser publicada: Cleise Cristine Ribeiro Borges Oliveira, Maiara da Silva Brandão Rodrigues, Carla Tatiane Oliveira Silva, Cláudia Silva Marinho Antunes Barros, Elieusa e Silva Sampaio e Cláudia Geovana da Silva Pires. 


\section{Referências}

1. Malta DC, Bernal RTI, Andrade SSCA, Silva MMA, Velasquez-Melendez G. Prevalência e fatores associados com hipertensão arterial autorreferida em adultos brasileiros. Rev Saude Pública. 2017;51(suppl 1):1-11. DOI: $10.1590 / \mathrm{s} 1518-8787.2017051000006$

2. Williams B, Mancia G, Spiering W, Agabiti Rosei E, Azizi M, Burnier M, et al. 2018 ESC/ESH Guidelines for the management of arterial hypertension. Eur Heart J. 2018 Sep;39(33):3021-104. DOI: 10.1093/ eurheartj/ehy339

3. Sociedade Brasileira de Cardiologia. 7th Brazilian Guideline of Arterial Hypertension. Arq Bras Cardiol [Internet]. 2016;107(Supl.3):1-83. DOI: http://dx.doi.org/10.5935/abc.20160152

4. Silva EC, Martins MSAS, Guimarães LV, Segri NJ, Lopes MAL, Espinosa MM. Prevalência de hipertensão arterial sistêmica e fatores associados em homens e mulheres residentes em município da Amazônia Legal. Rev Bras Epidemiol. 2016;19(1):38-51. DOI: 10.1590/1980-5497201600010004

5. Brasil. Ministério da Saúde. Secretaria de Vigilância em Saúde. Vigitel Brasil 2017. Vigilância de Fatores de Risco e Proteção para Doenças Crônicas por Inquérito Telefônico [Internet]. Brasília, DF; 2018. [cited 2019 Sep 5]. Available from: https://bvsms. saude.gov.br/bvs/publicacoes/vigitel_brasil_2017_ vigilancia_fatores_riscos.pdf

6. Cho KH, Lee SG, Nam SM, Lee EJ, Jang SY, Lee $\mathrm{SH}$, et al. Disparities in socioeconomic status and neighborhood characteristics affect allcause mortality in patients with newly diagnosed hypertension in Korea: a Nationwide cohort study, 2002-2013. Int J Equity Health. 2016;15(3):1-9. DOI: $10.1186 /$ s12939-015-0288-2

7. Jeon Y, Kim CR, Park J, Choi K, Kang MJ, Guk PS, et al. Health enequalities in hypertension and diabetes management among the poor in urban areas: a population survey analysis in south Korea. BMC Public Health. 2016;16(492):1-9. DOI: 10.1186/s12889-016-3169-8

8. Gupta R, Kaur M, Islam S, Mohan V, Mony P, Kumar R, et al. Association of Household Wealth Index Educational Status and Social Capital with Hypertension Awareness, Treatment, and Control in South Asia. Am J Hypertens. 2018;30(4):373-81. DOI: 10.1093/aih/hpw169
9. World Health Organization. Closing the gap in a generation. Health equity through action on the social determinants of healt. Comission on Social Determinants of Health, final report. Geneva; 2008.

10. Lee H. Socioeconomic disparities in the prevalence diagnosis, and control of hypertension in the context of a universal health insurance system. J Korean Med Sci. 2017;32:561-7. DOI: 10.3346/ jkms.2017.32.4.561

11. World Health Organization. Global Status Reporto on noncommunicable diseases 2014. Geneva; 2014.

12. Ribeiro AL, Duncan BB, Brant LC, Lotufo PA, Moinho JG, Barreto SM. Cardiovascular helth in Brazil: trend and perspectives. Circulation. 2016;133(4):422-33. DOI: 10.1161/ CIRCULATIONAHA.114.008727

13. World Health Organization. Plano de ação global para atividade física 2018-2030: mais pessoas ativas para um mundo mais saudável. Geneva; 2018.

14. Carvalho CJ, Marins JCB, Amorin PRS, Fernandes MS, Reis HHT, Sales SS, et al. Altas taxas de sedentarismo e fatores de risco cardiovascular em pacientes com hipertensão arterial resistente. Medicina (Ribeirão Preto). 2016; 49(2):124-33. DOI: 10.11606/issn.21767262.v49i2p124-133

15. Faleiro RC, Quaresma RS, Souza WM, Aquino ES. Avaliação do sedentarismo e risco de eventos cardiovasculares e sua correlação com o teste de caminhada de seis minutos. Sinapse Múltipla [Internet]. 2017 [cited 2019 Sep 1];6(2):139-53. Available from: http://periodicos.pucminas.br/ index.php/sinapsemultipla

16. Gillis EE, Sullivan JC. Sex difference in hypertension: recent advances. Hypertens. 2016; 68(6):1322-7. DOI: 10.1161/HYPERTENSIONAHA. 116.06602

17. Ostchega Y, Zhang G, Hughes JP, Nwankwo T. Factors Associated with hypertension control in US adults using 2017 ACC/AHA guidelines: National Health and Nutrition Examination Survey 19992016. Am J Hypertens. 2018;31(8):886-94. DOI: 10.1093/AJH/HPY047

18. Bosu WK, Aheto JMK, Zucchelli E, Reilly ST. Determinants of systemic hypertension in older adults in Africa: a systematic review. BMC Cardiovasc Disord. 2019;19(1):173. DOI: 10.1186/ s12872-019-1147-7 
19. Alves TA, Sousa BVN, Jesus VS, Camargo CL. Hipertensão arterial: conhecimento de jovens quilombolas. Rev Bras Saúde Func [Internet]. 2016 [cited 2019 Sep 1];1(1):39-49. Available from: http:// www.seer-adventista.com.br/ojs/index.php/RBSF/ article/view/713

20. Silva EC, Martins MSAS, Guimarães LV, Segri NJ, Lopes MAL, Espinosa, et al. Prevalência de hipertensão arterial sistêmica e fatores associados em homens e mulheres residentes em município da Amazônia Legal. Rev bras epidemiol. 2016;19(1):38-51. DOI: http://dx.doi. org/10.1590/1980-5497201600010004

21. Lobo LAC, Canuto R, Dias-da-Costa JS, Pattussi MP. Tendência temporal da prevalência de hipertensão arterial sistêmica no Brasil. Cad Saúde Pública. 2017;33(6):1-13. DOI: 10.1590/0102-311x00035316

22. Egan BM. Physical activity and hypertension. Knowing is not enough; we must apply. Willing is not enough; we must do-von goethe.
Hipertens. 2017;69:404-6. DOI: 10.1161/ HYPERTENSIONAHA.116.08508

23. Hines AL, Pollack CE, LaVeist TA, Jr Thorpe RJ. Race, Vigilant Coping Strategy, and Hypertension in na Integrated Community. Am J Hypertens. 2018;31(2):197-204. DOI: 10.1093/ajh/hpx164

24. Yi SS, Thorpe LE, Zanowiak JM, Trinh-Shevrin C, Islam NS. Clinical characteristics and lifestyle behaviors in a population-based sample of Chinese and South Asian immigrants with hypertension. Am J Hypertens. 2018;29(8):941-7. DOI: 10.1093/ ajhqhpw014

25. Hegde SM, Solomon SD. Influence of physical activity on hypertension and cardiac structure and function. Curr Hypertens Rep. 2015;17(10):1-13. DOI: $10.1007 / \mathrm{s} 11906-015-0588-3$

Recebido: 24 de setembro de 2019

Aprovado: 17 de outubro de 2019

Publicado: 23 de janeiro de 2020

A Revista Baiana de Enfermagem utiliza a Licença Creative Commons - Atribuição-NãoComercial 4.0 Internacional. https://creativecommons.org/licenses/by-nc/4.0/ Este artigo é de acesso aberto distribuído sob os termos da Licença Creative Commons (CC BY-NC). Esta licença permite que outros remixem, adaptem e criem a partir do seu trabalho para fins não comerciais. Embora os novos trabalhos tenham de lhe atribuir o devido crédito e não possam ser usados para fins comerciais, os usuários não têm de licenciar esses trabalhos derivados sob os mesmos termos. 